Bull. Korean Math. Soc. 48 (2011), No. 6, pp. 1125-1128

http://dx.doi.org/10.4134/BKMS.2011.48.6.1125

\title{
ON THE LOCAL COHOMOLOGY OF MINIMAX MODULES
}

\author{
AMIR MAFI
}

Abstract. Let $R$ be a commutative Noetherian ring, $\mathfrak{a}$ an ideal of $R$, and $M$ a minimax $R$-module. We prove that the local cohomology modules $H_{\mathfrak{a}}^{j}(M)$ are a-cominimax; that is, $\operatorname{Ext}_{R}^{i}\left(R / \mathfrak{a}, H_{\mathfrak{a}}^{j}(M)\right)$ is minimax for all $i$ and $j$ in the following cases: (a) $\operatorname{dim} R / \mathfrak{a}=1$; (b) $\operatorname{cd}(\mathfrak{a})=1$, where cd is the cohomological dimension of $\mathfrak{a}$ in $R$; (c) $\operatorname{dim} R \leq 2$. In these cases we also prove that the Bass numbers and the Betti numbers of $H_{\mathfrak{a}}^{j}(M)$ are finite.

\section{Introduction}

Throughout this paper, let $R$ denote a commutative Noetherian ring with non-zero identity and $\mathfrak{a}$ an ideal of $R$. For an $R$-module $M$, the $j$-th local cohomology module of $M$ with respect to $\mathfrak{a}$ is defined as

$$
H_{\mathfrak{a}}^{j}(M)=\underset{n}{\lim } \operatorname{Ext}_{R}^{j}\left(R / \mathfrak{a}^{n}, M\right) .
$$

We refer the reader to [6] or [9] for more details about local cohomology. Hartshorne [10] defined an $R$-module $M$ to be $\mathfrak{a}$-cofinite if $\operatorname{Supp}(M) \subseteq V(\mathfrak{a})$ and $\operatorname{Ext}_{R}^{i}(R / \mathfrak{a}, M)$ is finitely generated for all $i$ and asked:

For which rings $R$ and ideals $\mathfrak{a}$ are the modules $H_{\mathfrak{a}}^{j}(M) \mathfrak{a}$-cofinite for all $j$ and all finitely generated modules $M$ ?

With respect to this question, Hartshorne [10] showed that if $R$ is a complete regular local ring and $\mathfrak{a}$ is either a principal ideal or a prime ideal such that $\operatorname{dim} R / \mathfrak{a}=1$, then $H_{\mathfrak{a}}^{j}(M)$ is $\mathfrak{a}$-cofinite for all $j$ and for any finitely generated $R$-module $M$. Kawasaki [11] showed that, in general, for a finitely generated $R$-module $M$ if $\mathfrak{a}$ is principal, then the local cohomology modules $H_{\mathfrak{a}}^{j}(M)$ are $\mathfrak{a}$-cofinite. Delfino and Marley [7] proved that if $\mathfrak{a}$ is an ideal of an arbitrary local ring $R$ with $\operatorname{dim} R / \mathfrak{a}=1$ and $M$ a finitely generated $R$-module, then the modules $H_{\mathfrak{a}}^{j}(M)$ are $\mathfrak{a}$-cofinite (see also [14]). Recently, Bahmanpour and Naghipour [3] extended the results of Delfino and Marley [7] to arbitrary commutative Noetherian rings. Melkersson [13] showed that if $\operatorname{dim} R \leq 2$ and $M$

Received May 15, 2009.

2010 Mathematics Subject Classification. 13D45, 13E99.

Key words and phrases. local cohomology modules, minimax modules.

This research was in part supported by a grant from IPM (No. 900130064). 
a finitely generated $R$-module, then the modules $H_{\mathfrak{a}}^{j}(M)$ are $\mathfrak{a}$-cofinite. In [4] Belshoff, Slattery and Wickham studied the conditions under which the local cohomology modules $H_{\mathfrak{a}}^{j}(M)$ are almost cofinite. In fact, they showed that over a complete Gorenstein local domain $R$, if $\mathfrak{a}$ is an ideal of $R$ with $\operatorname{dim} R / \mathfrak{a}=1$ and $M$ is a Matlis reflexive module, then $\operatorname{Ext}_{R}^{i}\left(R / \mathfrak{a}, H_{\mathfrak{a}}^{j}(M)\right)$ is Matlis reflexive for all $i$ and $j$. Also, they obtained a couple of interesting results about the modules $H_{\mathfrak{a}}^{j}(M)$ and their Bass numbers (see also [5]). Kashyarmanesh and Khosh-Ahang [12] proved that if $R$ is a complete local ring and $M$ is a Matlis reflexive module, then $\operatorname{Ext}_{R}^{i}\left(R / \mathfrak{a}, H_{\mathfrak{a}}^{j}(M)\right)$ is Matlis reflexive for all $i$ and $j$ in the following cases: (a) $\operatorname{dim} R / \mathfrak{a}=1$; (b) $\mathfrak{a}$ is a principal ideal; (c) $\operatorname{dim} R \leq 2$. Azami, Naghipour and Vakili [1] defined an $R$-module $M$ is a-cominimax, as generalization of $\mathfrak{a}$-cofinite, if $\operatorname{Supp}(M) \subseteq V(\mathfrak{a})$ and $\operatorname{Ext}_{R}^{i}(R / \mathfrak{a}, M)$ is minimax for all $i$. In this paper, we eliminate the complete local hypothesis and improve the results of Kashyarmanesh and Khosh-Ahang [12]. In fact, if $R$ is an arbitrary commutative Noetherian ring and $M$ is a minimax module, then the modules $H_{\mathfrak{a}}^{j}(M)$ are $\mathfrak{a}$-cominimax whenever one of the above cases of the result of Kashyarmanesh and Khosh-Ahang holds.

\section{The results}

Zöschinger [16] introduced the interesting class of minimax modules and he has in [16] and [17] given many equivalent conditions for a module to be minimax. The $R$-module $M$ is said to be a minimax module if there is a finitely generated submodule $N$ of $M$ such that $M / N$ is Artinian. It was shown by Zink [15] and by Enochs [8] that a module over a complete local ring is minimax if and only if it is Matlis reflexive.

Remark 2.1. Let $M$ be an $R$-module.

(a) In view of [16] and [17], the class of minimax modules includes all finitely generated and all Artinian modules.

(b) Let $0 \longrightarrow N \longrightarrow M \longrightarrow L \longrightarrow 0$ be a short exact sequence of $R$ modules and $R$-homomorphisms. Then $M$ is minimax if and only if $N$ and $L$ are minimax, see [2].

(c) Every $\mathfrak{a}$-cofinite module is $\mathfrak{a}$-cominimax.

Lemma 2.2. Let $M$ be a minimax $R$-module. Then $\operatorname{Ext}_{R}^{i}\left(R / \mathfrak{a}, \Gamma_{\mathfrak{a}}(M)\right)$ and $\operatorname{Tor}_{i}^{R}\left(R / \mathfrak{a}, \Gamma_{\mathfrak{a}}(M)\right)$ is minimax for all $i$.

Proof. This is immediate by Remark 2.1 .

Lemma 2.3. Suppose that for any finitely generated $R$-module $N$ and for each $j \geq 0$, the local cohomology modules $H_{\mathfrak{a}}^{j}(N)$ are $\mathfrak{a}$-cofinite. Then for any minimax $R$-module $M$ and for each $j \geq 0$, the local cohomology modules $H_{\mathfrak{a}}^{j}(M)$ are $\mathfrak{a}$-cominimax. In fact, for each $j \geq 1$, the local cohomology modules $H_{\mathfrak{a}}^{j}(M)$ are $\mathfrak{a}$-cofinite. 
Proof. Since $M$ is minimax, there exists a short exact sequence

$$
0 \longrightarrow N \longrightarrow M \longrightarrow A \longrightarrow 0
$$

with $N$ a finitely generated module and $A$ an Artinian module. This induces the exact sequence

$$
0 \longrightarrow \Gamma_{\mathfrak{a}}(N) \longrightarrow \Gamma_{\mathfrak{a}}(M) \longrightarrow A \longrightarrow H_{\mathfrak{a}}^{1}(N) \longrightarrow H_{\mathfrak{a}}^{1}(M) \longrightarrow 0
$$

and for all $j \geq 2$, we have $H_{\mathfrak{a}}^{j}(M) \cong H_{\mathfrak{a}}^{j}(N)$. Hence, for all $j \geq 2, H_{\mathfrak{a}}^{j}(M)$ is $\mathfrak{a}$-cofinite. Let $L$ denote the kernel of the map $H_{\mathfrak{a}}^{1}(N) \longrightarrow H_{\mathfrak{a}}^{1}(M)$. Then $L$ is Artinian and so $\left(0:_{L} \mathfrak{a}\right)$ is finite length, since $A$ is Artinian and $\left(0:_{H_{\mathfrak{a}}^{1}(N)} \mathfrak{a}\right)$ is finitely generated. Thus, by $[13$, Proposition 4.1$] L$ is a-cofinite. From the exact sequence

$$
0 \longrightarrow L \longrightarrow H_{\mathfrak{a}}^{1}(N) \longrightarrow H_{\mathfrak{a}}^{1}(M) \longrightarrow 0
$$

we obtain the long exact sequence

$$
\cdots \rightarrow \operatorname{Ext}_{R}^{i}\left(R / \mathfrak{a}, H_{\mathfrak{a}}^{1}(N)\right) \rightarrow \operatorname{Ext}_{R}^{i}\left(R / \mathfrak{a}, H_{\mathfrak{a}}^{1}(M)\right) \rightarrow \operatorname{Ext}_{R}^{i+1}(R / \mathfrak{a}, L) \rightarrow \cdots
$$

By our hypothesis, $\operatorname{Ext}_{R}^{i}\left(R / \mathfrak{a}, H_{\mathfrak{a}}^{1}(N)\right)$ is finitely generated for all $i \geq 0$ and so $H_{\mathfrak{a}}^{1}(M)$ is $\mathfrak{a}$-cofinite. Hence $H_{\mathfrak{a}}^{j}(M)$ is $\mathfrak{a}$-cofinite for all $j \geq 1$.

Theorem 2.4. Let $M$ be a minimax $R$-module and suppose one of the following cases holds:
(a) $\operatorname{dim} R / \mathfrak{a}=1$;
(b) $\operatorname{cd}(\mathfrak{a})=1$;
(c) $\operatorname{dim} R \leq 2$.

Then $H_{\mathfrak{a}}^{j}(M)$ is $\mathfrak{a}$-cominimax. In fact, $H_{\mathfrak{a}}^{j}(M)$ is $\mathfrak{a}$-cofinite for all $j \geq 1$.

Proof. This follows by Lemma 2.3, [3, Corollary 2.7], [11, Lemma 2] and [13, Theorem 7.10].

Theorem 2.5. Let $M$ be a minimax $R$-module and suppose one of the following cases holds:
(a) $\operatorname{dim} R / \mathfrak{a}=1$;
(b) $\operatorname{cd}(\mathfrak{a})=1$;
(c) $\operatorname{dim} R \leq 2$.

Then $\operatorname{Tor}_{i}^{R}\left(R / \mathfrak{a}, H_{\mathfrak{a}}^{j}(M)\right)$ is minimax for all $j$ and $i$. In fact, $\operatorname{Tor}_{i}^{R}\left(R / \mathfrak{a}, H_{\mathfrak{a}}^{j}(M)\right)$ is finitely generated for all $i$ when $j \geq 1$.

Proof. This follows by Theorem 2.4, Lemma 2.2 and [13, Theorem 2.1].

Corollary 2.6. Let $M$ be a minimax $R$-module and suppose one of the following cases holds:
(a) $\operatorname{dim} R / \mathfrak{a}=1$;
(b) $\operatorname{cd}(\mathfrak{a})=1$;
(c) $\operatorname{dim} R \leq 2$.

Then the Bass numbers and the Betti numbers of $H_{\mathfrak{a}}^{j}(M)$ are finite for all $j$. 
Proof. This follows by Theorems 2.4 and 2.5 .

\section{References}

[1] J. Azami, R. Naghipour, and B. Vakili, Finiteness properties of local cohomology modules for a-minimax modules, Proc. Amer. Math. Soc. 137 (2009), no. 2, 439-448.

[2] K. Bahmanpour and R. Naghipour, On the cofiniteness of local cohomology modules, Proc. Amer. Math. Soc. 136 (2008), no. 7, 2359-2363.

[3] _ Cofiniteness of local cohomology modules for ideals of small dimension, J. Algebra 321 (2009), no. 7, 1997-2011.

[4] R. Belshoff, S. P. Slattery, and C. Wickham, The local cohomology modules of Matlis reflexive modules are almost cofinite, Proc. Amer. Math. Soc. 124 (1996), no. 9, 26492654.

[5] _ Finiteness properties for Matlis reflexive modules, Comm. Algebra 24 (1996), no. 4, 1371-1376.

[6] M. P. Brodmann and R. Y. Sharp, Local Cohomology: an algebraic introduction with geometric applications, Cambridge Studies in Advanced Mathematics, 60. Cambridge University Press, Cambridge, 1998.

[7] D. Delfino and T. Marley, Cofinite modules and local cohomology, J. Pure Appl. Algebra 121 (1997), no. 1, 45-52.

[8] E. Enochs, Flat covers and flat cotorsion modules, Proc. Amer. Math. Soc. 92 (1984), no. 2, 179-184.

[9] A. Grothendieck, Local Cohomology, Notes by R. Harthsorne, Lecture Notes in Math. 862, Springer-Verlag, 1966.

[10] R. Hartshorne, Affine duality and cofiniteness, Invent. Math. 9 (1970), 145-164.

[11] K. I. Kawasaki, Cofiniteness of local cohomology modules for principal ideals, Bull. London Math. Soc. 30 (1998), no. 3, 241-246.

[12] K. Khashyarmanesh and F. Khosh-Ahang, On the local cohomology of Matlis reflexive modules, Comm. Algebra 36 (2008), no. 2, 665-669.

[13] L. Melkersson, Modules cofinite with respect to an ideal, J. Algebra 285 (2005), no. 2, 649-668.

[14] K. I. Yoshida, Cofiniteness of local cohomology modules for ideals of dimension one, Nagoya Math. J. 147 (1997), 179-191.

[15] T. Zink, Endlichkeritsbedingungen für moduln über einem Noetherschen ring, Math. Nachr. 64 (1974), 239-252.

[16] H. Zöschinger, Minimax modules, J. Algebra 102 (1986), no. 1, 1-32.

[17] _ Über die Maximalbedingung für radikalvolle Untermoduh, Hokkaido Math. J. 17 (1988), no. 1, 101-116.

UNIVERSITY OF KURDISTAN

Pasdaran ST., P.O. Box: 416, SanandaJ, Iran

AND

SCHool of Mathematics

Institute for Research in Fundamental Science (IPM)

P.O. Box 19395-5746, TeHran, IrAN

E-mail address: a_mafi@ipm.ir 